\title{
All-optical logic gates based on nonlinear dielectric films
}

\author{
Dzedolik I.V., Lapayeva S.N. and Rubass A.F. \\ Taurida V. Vernadsky National University, 4 Vernadsky Ave., 95007 \\ Simferopol, Ukraine, e-mail: dzedolik@crimea.edu
}

Received: 15.04 .2008

\begin{abstract}
We analyze interaction of two nonlinear waves in thin amorphous dielectric film with reflecting edges. All-optical logic gates "AND", "OR" and "XOR", which are based on the dielectric film and involve no semiconductor components, are simulated both analytically and numerically.
\end{abstract}

Keywords: non-linear wave, dielectric film, optical logic gate

PACS: 42.30.-d, 42.65.-k, 42.70.Nq

UDC: 535.4

\section{Introduction}

Optical computing [1-3] has been proposed in the late 1980s, since the productivity of electronic computers does not satisfy modern requirements. Digital data can be transmitted to electronic processor by an optical fibre with the speed of light, but the maximum speed of switching of electronic logic gates is $50 \mathrm{ps}\left(2 \times 10^{10} \mathrm{~Hz}\right)$ for the average power $0.5 \mathrm{~mW}$ (the energy $25 \mathrm{fJ}$ ) per one switching [4]. It is known that the switching speed of logic gates based on semiconductors is restricted by capacitance of $p-n$ junctions.

Modern semiconductor logic gates have small sizes $(<0.1 \mu \mathrm{m})$, though their switching is limited by interlinking capacitance $\left(>10^{-10} \mathrm{~s}\right)$ [4]. At the same time, the switching speed of optical logic gates is limited only by the velocity of light passing through it ( $\sim 3 \mathrm{fs}$ or $3 \times 10^{14} \mathrm{~Hz}$ ). All-optical logic gates have the sizes close to the light wavelength $(\sim 1 \mu \mathrm{m})$, thus decreasing the packing density of logic gates on the chip. An alternative way for creating high-speed computers with great productivity and small sizes lies in combining optical data bus with a quantum processor [5].

However, the problem of commutation of optical signals appearing in the area of information technologies has not been solved completely. The all-optical logic gates based on linear and nonlinear photonic crystals [6-14] are now extensively developed. The mentioned optical technologies allow one to conduct the processing under the conditions when the optical signals propagate through devices at the speed of light. 
A great attention has once been given to the optical logic gates based on bistable properties of nonlinear media $[15,16]$. However, the bistable effects depend on the relaxation time of medium and produce a delay of logic-gate function. Later on, the logic gates based on the Kerr effect [17-19] and two-photon light scattering in crystals [20, 21] have been suggested. The optical logic gates based on polarization systems [22, 23] may also be considered. Such the logic gates require highly coherent input signals and, moreover, their initial phase difference must be constant, since the dynamics of wave polarization is due to interference effects in the gates. In particular, the logic gates based upon ferroelectric liquid crystals [24], liquid-crystalline cells [25, 26], silicon micro-ring resonators [27, 28] and local nonlinear Mach-Zehnder interferometer [29] have been proposed. Today the main attention is focused on quantum logic gates and quantum computing [30-41]. This is because the quantum logic gates have minimal sizes.

The logic gates based on the liquid crystals are relatively slow, whereas the photonic crystals or other optical elements are very complicated in production. The problems of stability of quantum states and their non-destructive control have not yet been solved. However, we should take into account a fundamental limit for the optical elements [42], because we focus on the computing processes.

That is why we suggest the other optical logic gates that operate at high speeds and have small sizes [43-45]. Our logic gates are associated with the Kerr effect in thin amorphous dielectric films and, moreover, they are simple enough for producing. Both periodic (cnoidal) and solitary nonlinear waves can possibly appear in a dielectric medium, depending on the relations of parameters of the medium and the electromagnetic field $[6,13]$. Nonlinear spatial waves appear if the medium is placed into resonator. A dielectric film with plane-parallel reflecting edges could be represented as a Fabry-Perot resonator $[6,16]$. As a consequence of nonlinear interaction of the waves, there are minima and maxima of the field intensity at the mirrors of this dielectric resonator. A presence of a minimum or maximum field at a given point of output resonator mirror can be treated as a logic signal (zero or one) in an optical logic gate.

The purpose of the present work is simulating the optical logic gates based on the thin amorphous dielectric films, which manifest almost no absorption for the electromagnetic waves.

\section{Nonlinear Modes of Dielectric Resonator}

We consider a physical mechanism of interaction of two electromagnetic waves injected into an amorphous dielectric film. The dielectric film with the thickness $h$ and flat semitransparent reflecting edges represents a one-dimensional dielectric resonator [11-13].

In Fig. 1 we show a scheme of the resonator, where $M_{1}$ and $M_{2}$ are the reflecting edges (i.e., semi-transparent mirrors of the resonator), $A$ and $B$ the wedged input waveguides and $Z$ the output planar waveguide. All the waveguides have a doped interior layer with the refraction index $n_{1}$ and quartz shells with the index $n_{0}\left(n_{1}>n_{0}\right)$. The interaction of two nonlinear waves takes place in the planar dielectric Fabry-Perot 
resonator with the refraction index $n_{1}$, the shell with the index $n_{0}$ and the semitransparent mirrors $M_{1}$ and $M_{2}$. The waveguides $A, B$, on the one side, and the waveguide $Z$, on the other side, are attached respectively to the input mirror $M_{1}$ and the output mirror $M_{2}$ of the resonator. The shell thickness of the joint input and output waveguides is equal to the thickness of the resonator shell. The waveguides $A$ and $B$ represent inputs, through which the optical signals are injected into the resonator, while the waveguide $Z$ is an output, through which the optical signal leaves the system [43-45]. The input waveguides $A$ and $B$ have wedge tapered profiles that transform into flat one, with the cross section equal to that of the waveguides. The profile of the output waveguide is also flat, with the same cross section. Like a diffraction grating, the input mirror $M_{1}$ has some gaps along $y$-direction. This is done for elimination of phase difference of the input signals. The optical signals with the electric component $E_{y}$ of the same $y$-polarization (TE modes) propagate parallel beginning from the input mirror $M_{1}$ of the resonator. Then the output signal leaves through the mirror $M_{2}$ and excites the TE mode in the output waveguide. We suppose that the signal modes do not change in the tapered waveguides, since their profiles vary slowly.

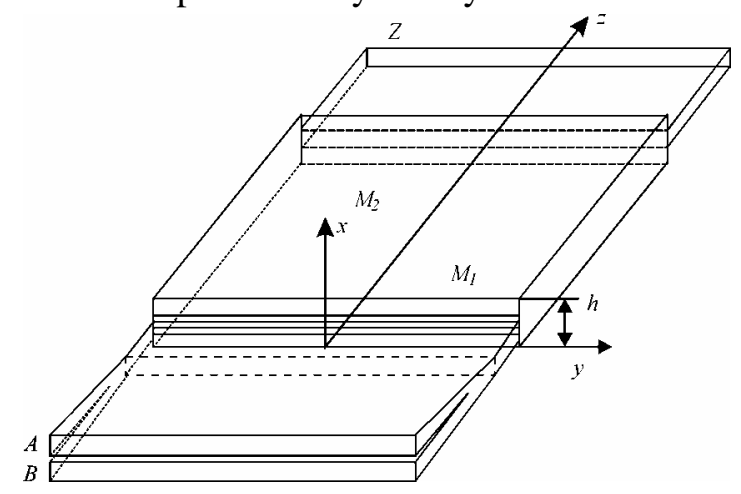

Fig. 1. Flat dielectric resonator based on thin film (shells are not depicted).

The dielectric permittivity of the resonator is $\varepsilon=1+4 \pi \chi_{1}(\omega)+4 \pi \chi_{3} I$, where $\chi_{1}(\omega)$ denotes the linear susceptibility of the medium, $\chi_{3}$ its cubic susceptibility and $I \sim|E|^{2}$ the intensity of the electromagnetic field. The input power must be sufficiently high for the nonlinear Kerr effect to appear in the medium. The nonlinear effect appears in the glass with multi-component additive once the input power exceeds $1 \mathrm{~W}$. The nonlinear response of the glass takes a few femtoseconds to become steady [6].

We consider the optical signals as quasi-monochromatic electromagnetic waves $(\Delta \omega<<\omega)$ that can be described in the film by only one transverse electric component $E_{y}=e_{y}(x) \exp \left(i \omega t-i k_{\ell} z+i \phi_{0}\right)=e_{y} e^{i \phi}(\partial / \partial y \rightarrow 0)$ and two magnetic components $B_{x}=-\frac{c k_{z}}{\omega} E_{y}$ and $B_{z}=-i \frac{c}{\omega} \frac{d E_{y}}{d x}$. The square of the wave vector of $\ell$ th resonator 
mode is equal to $k_{\ell}^{2}=k_{x \ell}^{2}+k_{z \ell}^{2}$, where $k_{x \ell}$ means the transverse component of the wave vector, $k_{z \ell}=\ell \pi / L$ the propagation constant of the mode traversing along the longitudinal axis, $L$ the resonator length, $\ell= \pm 1, \pm 2, \ldots$ and $\phi_{0}$ is the initial signal phase at the input.

The equation for the electric field, $\left(\nabla^{2}-c^{-2} \varepsilon \partial^{2} / \partial t^{2}\right) E_{y}=0$, looks like

$$
\frac{d^{2} e_{y}}{d x^{2}}+q_{1} e_{y} \pm q_{2} e_{y}^{3}=0
$$

where $\bar{x}=x \omega / c, q_{1}=1+4 \pi \chi_{1}-\ell^{2} c^{2} \pi^{2} / \omega^{2} L^{2}, q_{2}=4 \pi\left|\chi_{3}\right|$ and the signs plus and minus correspond respectively to focusing and defocusing nonlinear media. After normalizing $\left(e_{y}=\sqrt{q_{1} / q_{2}} f(X)\right.$, with $\left.X=\bar{x} \sqrt{q_{1}}\right)$ Eq. (1) takes the following form:

$$
\frac{d^{2} f}{d X^{2}}+f \pm f^{3}=0
$$

Let us assume that only one nonlinear $\ell$ th mode is excited in the resonator. For that mode we rewrite Eq. (2) for the case of defocusing medium as $\frac{1}{2} \frac{d}{d X}\left(\frac{d f}{d X}\right)^{2}+\left(f-f^{3}\right) \frac{d f}{d X}=0$ and find its solution by using the elliptic integral $X=\int_{f_{0}}^{f} \frac{d f}{\left[a_{0}^{2}-f^{2}+f^{4} / 2\right]^{1 / 2}}, \quad$ where $\quad a_{0}^{2}=(d f / d X)_{0}^{2}+f_{0}^{2}-f_{0}^{4} / 2 . \quad$ Then we put $2 a_{0}^{2}-2 f^{2}+f^{4}=\left(b_{1}^{2}-f^{2}\right)\left(b_{2}^{2}-f^{2}\right), \quad$ where $b_{1}^{2} b_{2}^{2}=2 a_{0}^{2}, \quad b_{1}^{2}+b_{2}^{2}=2, \quad f_{0}=0 \quad$ and $0<f<b_{2}<b_{1}$. As a result, we obtain $X=\frac{\sqrt{2}}{b_{1}} F\left[\arcsin \left(\frac{f}{b_{2}}\right), \tilde{k}\right]$ for $\tilde{k}=b_{2} / b_{1}$. Now we convert the latter relation and finally get the spatial cnoidal wave:

$$
f=b_{2} \operatorname{sn}\left(\sqrt{b_{1}^{2} / 2} X, \tilde{k}\right) \text {, }
$$

where $\operatorname{sn}(X, \tilde{k})$ is the Jacobi elliptic sine function. The cnoidal wave has its maxima and minima at the resonator mirrors.

Let us estimate the distance between the zeros $x_{0}=\sqrt{2} / b_{1}$ of the spatial wave with the intensity $f^{2}$. For the laser radiation with the frequency $\omega \sim 10^{15} \mathrm{~s}^{-1}$ propagating in the medium with the parameters $\varepsilon_{1}=1.5$ and $\chi_{3} \sim 10^{-20} \mathrm{~cm}^{3} / \mathrm{erg}$, we obtain $x_{0} \sim 10^{-5}$. This distance can be as small as the Heisenberg uncertainty principle allows 
$\left(\Delta x \sim 1 / \Delta k_{x}\right)$.

Now we have the equation for the two $\ell$ th modes excited in the resonator, which have the equal frequencies $\omega$ and the wave vectors $k_{z \ell}$ in the defocusing medium:

$$
\begin{array}{r}
\left\{\frac{d^{2}}{d \bar{x}^{2}}+q_{1}-q_{2}\left[e_{y 1}^{2}+e_{y 2}^{2}+2 e_{y 1} e_{y 2} \cos \left(\phi_{01}-\phi_{02}\right)\right]\right\} \times \\
\left(e_{y 1} \exp \left(i \phi_{01}\right)+e_{y 2} \exp \left(i \phi_{02}\right)\right)=0 .
\end{array}
$$

Since the initial phases $\phi_{01} \neq \phi_{02}$ are arbitrary, we can equate to zero each of the terms in Eq. (4) that include $\exp \left(i \phi_{0 j}\right)$. Then we derive the combined equations

$$
\begin{aligned}
& \frac{d^{2} f_{1}}{d X^{2}}+f_{1}-\left[f_{1}^{2}+f_{2}^{2}+2 f_{1} f_{2} \cos \left(\phi_{01}-\phi_{02}\right)\right] f_{1}=0, \\
& \frac{d^{2} f_{2}}{d X^{2}}+f_{2}-\left[f_{1}^{2}+f_{2}^{2}+2 f_{1} f_{2} \cos \left(\phi_{01}-\phi_{02}\right)\right] f_{2}=0 .
\end{aligned}
$$

The initial phase difference $\phi_{01}-\phi_{02}$ affects the values of the functions $f_{1}$ and $f_{2}$ obtained from the combined Eqs. (5). This is because the cosine values for each pair of the input signals change arbitrarily from -1 to 1 . However, the gaps at the input mirror (see Fig. 1) eliminate the phase difference for the main diffraction maximum if the signals pass the resonator through the diffraction grating, i.e. we have $\phi_{01}-\phi_{02}=0$.

We have derived numerical solutions of the system of Eqs. (5) for the functions $f_{1}$ and $f_{2}$ with the cosine value equal to unity in the case of identical boundary conditions for the waves. The wave intensities $I \sim f_{1}^{2}, f_{2}^{2}$ are represented in Fig. 2 and Fig. 3. As follows from our numerical solutions of Eqs. (5), the spatial waves are generated in the dielectric film resonator and their field maxima and zeros are located at the output resonator mirror.

Notice that the units taken for the intensity, the thickness $h$ of the film and the coordinate $X$ are normalized and depend upon the parameters of both the film and the waves. For example, the normalized unit used for the coordinate $X$ or the film thickness $h$ is equal to $0.7 \mu \mathrm{m}$ for the dielectric film with the permittivity $\varepsilon_{1}=1+4 \pi \chi_{1}=1.96$,

$4 \pi \chi_{2}=10^{-10} \mathrm{~m} / \mathrm{V}$, the wave mode $\ell=10$ of the resonator, the resonator length $L=0.75 \mu \mathrm{m}$ and the light wavelength $\lambda=1.55 \mu \mathrm{m}$.

\section{Optical Logic Gates}

The presence or absence of the field maxima (or zeros) at the output mirror along its transverse axis $x$ allows realizing optical logic gates if only the spatial waves are excited. 


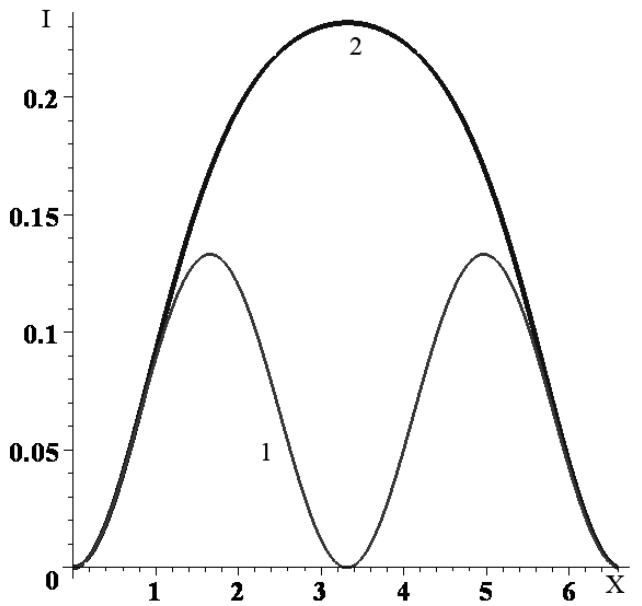

Fig. 2. Intensities of the waves for the cases of one signal ( $A$ or $B$ - see curve 1) and two signals ( $A$ and $B$ - see curve 2). The film thickness is $h=6.6$ and the wave boundary conditions $f_{1}(0)=0$ $f_{2}(0)=0, \quad d f_{1}(0) / d X=0.3526 \quad$ and $d f_{1}(0) / d X=0.3526 \quad$ (all units are normalized).

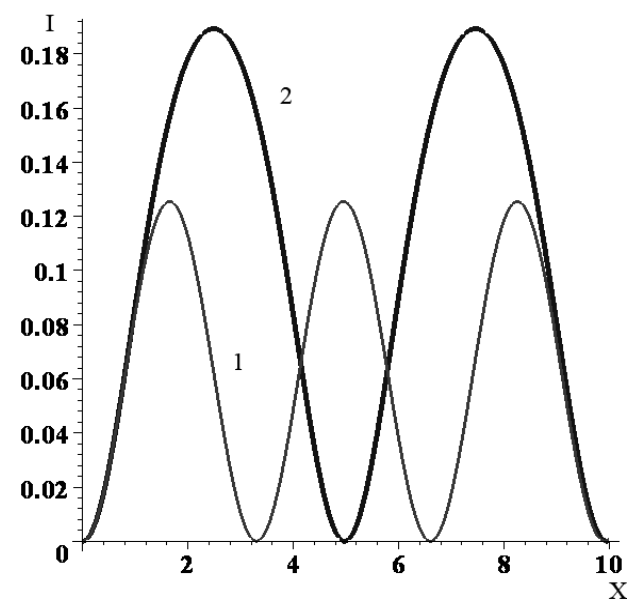

Fig. 3. Intensities of the waves for the cases of one signal ( $A$ or $B$ - see curve 1) and two signals ( $A$ and $B$ - see curve 2). The film thickness is $h=10.0$ and the wave boundary conditions $f_{1}(0)=0$, $f_{2}(0)=0, \quad d f_{1}(0) / d X=0.343 \quad$ and $d f_{1}(0) / d X=0.343 \quad$ (all units are normalized).

The optical signals are injected into the input waveguides $A$ and $B$ of the logic gate (see Fig. 1) and the output signal is received at the waveguide $Z$.

The logic gate "AND" with the positive logic (Table 1) has been simulated for the dielectric film with the normalized thickness $h=6.6$. In Table 1 the logic one and logic zero are marked respectively as ' 1 ' and ' 0 '. The film thickness is chosen such that we obtain zeros on the upper and lower surfaces (see Fig. 2).

Table 1. Logic gates with the positive logic.

The output waveguide $Z$ with the

\begin{tabular}{|c|c|c|c|}
\hline $\begin{array}{c}\text { Logic } \\
\text { gate }\end{array}$ & $\begin{array}{c}\text { Input } \\
\boldsymbol{A}\end{array}$ & $\begin{array}{c}\text { Input } \\
\boldsymbol{B}\end{array}$ & $\begin{array}{c}\text { Output } \\
\boldsymbol{Z}\end{array}$ \\
\hline \multirow{4}{*}{ AND } & 0 & 0 & 0 \\
\cline { 2 - 4 } & 1 & 0 & 0 \\
\cline { 2 - 4 } & 0 & 1 & 0 \\
\cline { 2 - 4 } & 1 & 1 & 1 \\
\hline \multirow{4}{*}{ OR } & 0 & 0 & 0 \\
\cline { 2 - 4 } & 1 & 0 & 1 \\
\cline { 2 - 4 } & 0 & 1 & 1 \\
\cline { 2 - 4 } & 1 & 1 & 1 \\
\hline \multirow{4}{*}{ XOR } & 0 & 0 & 0 \\
\cline { 2 - 4 } & 1 & 0 & 1 \\
\cline { 2 - 4 } & 0 & 1 & 1 \\
\cline { 2 - 4 } & 1 & 1 & 0 \\
\hline
\end{tabular}
normalized thickness $h_{z}=1.4$ must be attached to the output mirror $M_{2}$. The $y$-axis of the waveguide $Z$ should cross the point $X=3.3$ (see Fig. 2). The output signal intensity is detected on the level of $I \sim 0.1$. For the boundary conditions $\quad f_{1}(0)=0 \quad$ and $d f_{1}(0) / d X=0.3526$, we have the signal intensity $I \sim f_{1}^{2}$ (curve 1 in Fig. 2), if one of the signals (that in the waveguide $A$ or $B$ ) is present and the signal in the other waveguide is absent. Then the signal in the output waveguide $Z$ is 
absent (see Fig. 4). If both of the input signals $A$ and $B$ are present and the same boundary conditions are true $\left(f_{1}(0)=0, f_{2}(0)=0, \frac{d f_{1}(0)}{d X}=0.3526\right.$ and $\left.\frac{d f_{2}(0)}{d X}=0.3526\right)$, we obtain the identical intensities of the signals $f_{1}^{2}$ and $f_{2}^{2}$ (curve 2 in Fig. 2). Then we have the signal in the output waveguide $Z$ shown in Fig. 5. For our model of the gate "AND", the signal-to-noise ratio in the output waveguide is $6.6 \mathrm{~dB}$.

The logic gate "OR" with the positive logic (see Table 1) can be produced if the two input waveguides $A$ and $B$ are joined with the output waveguide $Z$, which has a wedge profile.

Finally, the logic gate "XOR" with the positive logic (see Table 1) has been simulated by the dielectric film with the normalized thickness $h=10.0$. The output waveguide $Z$ (Fig. 1) with the normalized thickness 1.4 should be attached to the output mirror $M_{2}$ and the $y$-axis of the waveguide must cross the point $X=5.0$ (see Fig. 3). The signal intensity $f_{1}^{2}$ (curve 1 in Fig. 2) occurs for the boundary conditions $f_{1}(0)=0$ and
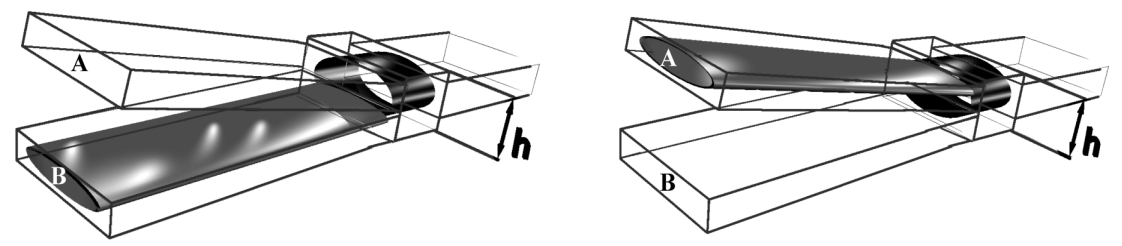

Fig. 4. Scheme of the optical logic gate "AND": only one of the input signals $A$ and $B$ is present.

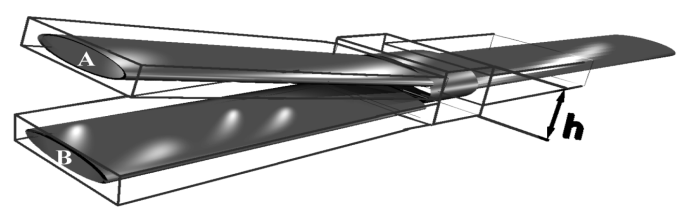

Fig. 5. Scheme of the optical logic gate "AND": both the input signals $A$ and $B$ are present.

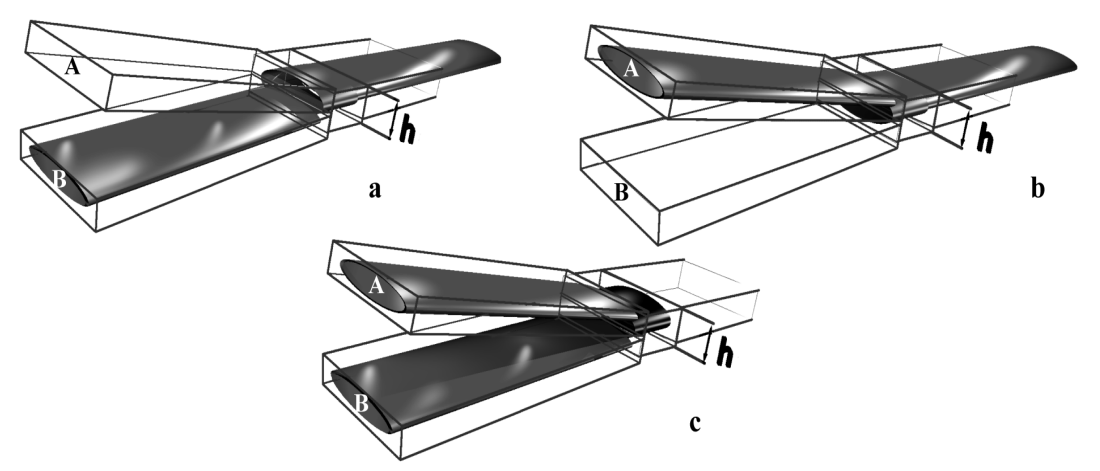

Fig. 6. Scheme of the optical logic gate "XOR": (a) input signal $A$, (b) input signal $B$, and (c) both input signals $A$ and $B$ are present. 
$\frac{d f_{1}(0)}{d X}=0.343$, if one of the signals (that referring to the waveguide $A$ or $B$ ) is present and the signal in the other waveguide is absent. Then the signal appears in the output waveguide $Z$. If both of the input signals $A$ and $B$ are present and the boundary conditions remain to be same $\left(f_{1}(0)=0, f_{2}(0)=0, \frac{d f_{1}(0)}{d X}=0.343\right.$ and $\left.\frac{d f_{2}(0)}{d X}=0.343\right)$, we have the identical signal intensities $f_{1}^{2}$ and $f_{2}^{2}$ (see curve 2 in Fig. 3). The field intensity is detected on the level of $I \sim 0.1$. For our model of the gate "XOR", the signal-to-noise ratio in the output waveguide is $4 \mathrm{~dB}$. Functioning of the logic gate "XOR" is illustrated in Fig. 6. If the power of the input signals arises, the signal-to-noise ratio increases because the spatial profile becomes sharper.

\section{Conclusion}

Thus, we have shown that there is a possibility for creating easily produced logic gates "AND", "OR" and "XOR" of rather small sizes, when basing on the thin amorphous dielectric films. The construction of our logic gates does not involve any semiconductor components, etc. If nonlinear spatial waves are excited in the film, the speed of the logic gate operation is determined by the relaxation time of the nonlinear response for a given dielectric medium. The latter is about few femtoseconds. This means that the all-optical logic gates suggested by us can use femtosecond pulses as signals. We have also shown how it is possible to conduct all the logic operations with the optical signals, using the optical logic gates "AND", "OR" and "XOR" mentioned above.

\section{References}

1. Feitelson D G. Optical computing: a survey for computer scientists. Cambridge: MIT Press (1988).

2. Arrathoon R, Ed. Optical computing: digital and symbolic. New York: Marcel Deccer (1989).

3. McAulay A D. Optical computer architectures: the application of optical concepts to next generation computer. New York: John Wiley \& Sons (1991).

4. Meindl J D, 1995. Low power microelectronics: retrospect and prospect. Proc. IEEE. 83: 619-635.

5. Nielsen MA and Chang I L. Quantum computation and quantum information. Cambridge: Cambridge Univ. Press (2000).

6. Kivshar Yu S and Agrawal G P. Optical Solitons: From Fibers to Photonic Crystals. Academic Press (2003).

7. Cuesta-Soto F, Martinez A, Garcia J, Ramos F, Sanchis P, Blasco J and Marti J, 2004. All-optical switching structure based on a photonic crystal directional coupler. Opt. Exp. 12(1): 161-167.

8. Notomi M, Shinya A, Mitsugi S, Kira G, Kuramochi E and Tanabe T, 2005. Optical bistable switching action of Si high-Q photonic-crystal nanocavities. Opt. Exp. 13(7): 2678-2687. 
9. Zhu Z-H, Ye W-M, Ji J-R, Yuan X and Zen C, 2006. High-contrast light-by-light switching and AND gate based on nonlinear photonic crystals. Opt. Exp. 14(5): 1783-1788.

10. Fujisawa T and Koshiba M, 2006. All-optical logic gates based on nonlinear slotwaveguide couplers. J. Opt. Soc. Am. B. 23(4): 684-691.

11. Dzedolik I V, 2007. Optical logic gate 'AND', controllable photon crystal. IEEE Catalog No. 07EX1829C. ISBN 1-4244-1322-2. Library of Congress. 2007927064: 23.

12. Dzedolik I V, 2007. One-dimensional controllable photonic crystal. J. Opt. Soc. Am. B 24(10): 2741-2745.

13. Dzedolik I V. Polaritons in optical fibers and dielectric resonators. Simferopol: DIP (2007).

14. Zou H, Liang G-Q and Wang H-Z, 2008. Efficient all-optical dual-channel switches, logic gates, half-adder, and half-subtracter in a one-dimensional photonic heterostructure. J. Opt. Soc. Am. B. 25(3): 351-360.

15. Walker A C, 1986. Application of bistable optical logic gate arrays to all-optical digital parallel processing. Appl. Opt. 25(10): 1578-1585.

16. Craig R G, Buller G S, Tooley F A P, Smith S D, Walker A C and Wherrett B S, 1990. All-optical programmable logic gate. Appl. Opt. 29(14): 2148-2152.

17. Normandin R, 1986. All-optical, fiber-optic modulator and logic gate using nonlinear refraction and absorption. Opt. Lett. 11(11): 751-753.

18. Bhardwaj A, Hedekvist P O and Vahala K, 2001. All-optical logic circuits based on polarization properties of nondegenerate four-wave mixing. J. Opt. Soc. Am. B. 18(5): 657-665.

19. Scheuer $\mathbf{J}$ and Orenstein $\mathrm{M}$, All-optical gates facilitated by soliton interactions in a multilayered Kerr medium. J. Opt. Soc. Am. B. 22(6): 1260-1267.

20. Shcherbakov A S, Tepichin Rodriguez E and Aguirre López A, 2004. All-optical performing of logic-based operations due to a two-photon light scattering. Revista Mexicana de Fisica. 50(2): 140-148.

21. Puddu E, Allevi A and Andreoni A, 2004. All-optical logic operations by means of two interlinked $\chi^{(2)}$ interactions in a single crystal. J. Opt. Soc. Am. B. 21(19): 1839-1847.

22. Zaghloul Y A and Zaghloul A R M, 2006. Unforced polarization-based optical implementation of binary logic. Opt. Exp. 14(16): 7252-7269.

23. Zaghloul Y A and Zaghloul A R M, 2006. Complete all-optical processing polarization-based binary logic gates and optical processors. Opt. Exp. 14(21): 9879-9895.

24. Handschy M A, Johnson K M, Gathey W T and Pagano-Stauffer L A, 1987. Polarization-based optical parallel logic gate utilizing ferroelectric liquid crystals. Opt. Lett. 12(8): 611-613.

25. Takizawa K, Kikuchi H, Aida T and Okada M, 1989. Optical parallel logic gate using a Pockels readout optical modulator and twisted nematic liquid-crystal cells. Opt. Lett. 14(4): 208-210.

26. Wang Y-Y, Chen J-Y and Chen L-W, 2007. Optical switches based on partial band gap and anomalous refraction in photonic crystals modulated by liquid crystals. Opt. 
Exp. 15(16): 10033-10040.

27. Zhao C, Zhang X, Liu H, Liu D and Huang D, 2005. Tunable all-optical NOR gate at $10 \mathrm{~Gb} / \mathrm{s}$ based on SOA fiber ring laser. Opt. Exp. 13(8): 2793-2797.

28. Xu Q and Lipson M, 2007. All-optical logic gates based silicon micro-ring resonators. Opt. Exp. 15(3): 924-929.

29. Wu Y-D, Shih T-T and Chen M-H, 2008. New all-optical logic gates based on the local nonlinear Mach-Zehnder interferometer. Opt. Exp. 16(1): 248-257.

30. Stockton J, Armen M and Mabuchi H, 2002. Programmable logic devices in experimental quantum optics. 19(12): 3019-3027.

31. Valiev K A, 2005. Quantum computers and quantum computing. Usp. Fiz. Nauk. 175(1): 1-39.

32. Milburn G, 2007. Quasi-probability methods for multimode conditional optical gates. J. Opt. Soc. Am. B. 24(2): 167-170.

33. White A G, Gilchrist A, Pryde G J, O'Brien J L, Bremner M J and Langford N K, 2007. Measuring two-qubit gates. J. Opt. Soc. Am. B. 24(2): 172-183.

34. Kieling K, Gross D and Eisert J, 2007. Minimal resources for linear optical one-way computing. J. Opt. Soc. Am. B. 24(2): 184-188.

35. Berry D W, Lvovsky A I and Sanders B C, Efficiency limits for linear optical processing of single photons and single-rail qubits. J. Opt. Soc. Am. B. 24(2): 189-197.

36. Utsunomiya S, Master C P, Yamamoto Y, 2007. Algorithm-based analysis of collective decoherence in quantum computation. J. Opt. Soc. Am. B. 24(2): 198-208.

37. Franson J D, Pittman TB and Jacobs BC, 2007. Zeno logic gates using microcavities. J. Opt. Soc. Am. B. 24(2): 209-213.

38. Soudagar Y, Bussieres F, Berlin G, Lacroix S, Fernandez J M and Godbout N, 2007. Cluster-state quantum computing in optical fibers. J. Opt. Soc. Am. B. 24(2): 226230.

39. Barak R and Ben-Aryeh Y, 2007. Quantum fast Fourier transform and quantum computation by linear optics. J. Opt. Soc. Am. B. 24(2): 231-240.

40. Guney D O and Meyer D A, 2007. Creation of entanglement and implementation of quantum logic gate operations using a three-dimensional photonic crystal singlemode cavity. J. Opt. Soc. Am. B. 24(2): 283-294.

41. Deng L-P, Wang H and Wang K, 2007. Quantum CNOT gates with orbital angular momentum and polarization of single-photon quantum logic. J. Opt. Soc. Am. B 24(9): 2517-2520.

42. Muller D A B, 2007. Fundamental limit for optical components. J. Opt. Soc. Am. B. 24(19): A1-A18.

43. Dzedolik I V, 2007. Optical logic gate "XOR", Patent of Ukraine. G02F 3/00 No 26823.

44. Dzedolik I V, 2007. Optical logic gate “AND”, Patent of Ukraine. G02F 3/00 No 27194.

45. Dzedolik I V, 2007. Optical logic gate "OR", Patent of Ukraine. G02F 3/00 No 27196. 